\title{
Tratamento cirúrgico conservador de fraturas múltiplas da face após queda
}

\author{
Conservative surgical treatment of multiple fractures of the face after a fall \\ Tratamiento quirúrgico conservador de múltiples fracturas del rostro tras una caída
}

Recebido: 12/01/2022 | Revisado: 16/01/2022 | Aceito: 21/01/2022 | Publicado: 23/01/2022

\author{
Heleninha dos Santos Galvão \\ ORCID: https://orcid.org/0000-0002-4977-1610 \\ Universidade Federal de Mato Grosso do Sul, Brasil \\ E-mail: heleninha1997@gmail.com \\ Julio Cesar Leite da Silva \\ ORCID: https://orcid.org/0000-0002-9835-7432 \\ Universidade Federal de Mato Grosso do Sul, Brasil \\ E-mail: heleninha1997@gmail.com \\ José Carlos Garcia de Mendonça \\ ORCID: https://orcid.org/0000-0003-3217-872X \\ Universidade Federal de Mato Grosso do Sul, Brasil \\ E-mail: jose.mendonca@ufms.br \\ Gustavo Silva Pelissaro \\ ORCID: https://orcid.org/0000-0003-3475-6001 \\ Universidade Federal de Mato Grosso do Sul, Brasil. \\ E-mail: gustavopelissaro@hotmail.com \\ Janayna Gomes Paiva-Oliveira \\ ORCID: https://orcid.org/0000-0003-2090-2872 \\ Universidade Federal do Mato Grosso do Sul, Brasil \\ E-mail:jgpaivaoliveira@gmail.com \\ Francielly Thomas Figueiredo \\ ORCID: https://orcid.org/0000-0003-0196-1172 \\ Universidade Federal do Mato Grosso do Sul, Brasil \\ E-mail: francielly.thomas@ufms.br \\ Alana Oswaldina Gavioli Meira dos Santos \\ ORCID: https://orcid.org/0000-0002-5591-5262 \\ Universidade Federal de Mato Grosso do Sul, Brasil \\ E-mail: nagavioli@gmail.com \\ Athilla Arcari Santos \\ ORCID: https://orcid.org/0000-0003-1977-2521 \\ Universidade Federal do Mato Grosso do Sul, Brasil \\ E-mail: athilla.arcari@ufms.br \\ Ellen Cristina Gaetti-Jardim \\ ORCID: https://orcid.org/0000-0003-2471-465X \\ Universidade Federal de Mato Grosso do Sul, Brasil \\ E-mail: ellen.jardim@ufms.br
}

\begin{abstract}
Resumo
As fraturas faciais configuram um problema de saúde pública em virtude da necessidade de atenção especializada, ambiente adequado e recursos financeiros. Apresentam causas variadas, sendo que acidentes desportivos, de trabalho, de trânsito e quedas se destacam como as principais, sendo as mesmas relacionadas a idade, sexo do paciente e direção do trauma. Este trabalho tem como objetivo relatar o caso clínico de um paciente do sexo masculino vítima de queda de 4,5 metros de altura, impelindo a face contra as bordas de uma piscina. Atendido no Hospital Universitário Maria Aparecida Pedrossian, necessitando de atendimento ambulatorial e cirúrgico para tratamento de fraturas múltiplas da face. Ao exame físico inicial apresentou edema em terço médio e inferior da face, acuidade visual e motilidade ocular preservadas, mobilidade em parassínfise, laceração em lábio inferior, mobilidade acentuada em elementos dentais, fragmentos de tecido ósseo bucal exposto em região de pré-maxila, ausências dentais e movimentos mandibulares alterados. Na tomografia computadorizada, mostrou fratura cominutiva em região de maxila anterior, fratura bilateral de cabeça da mandíbula e fratura em parassínfise mandibular do lado esquerdo. Assim, o tratamento de eleição foi o cirúrgico sob anestesia geral para colocação da barra de Erich e bloqueio maxilomandibular, redução e fixação de fratura anterior de mandíbula e remoção dos fragmentos ósseos e dentes sem suporte em região de maxila. Nos controles pósoperatórios o paciente se manteve sem queixas após a remoção do bloqueio, com movimentos mandibulares preservados e retorno às funções mastigatórias. Após seis meses do trauma, o paciente encontra-se totalmente reabilitado.
\end{abstract}

Palavras-chave: Fraturas ósseas; Terapêutica; Resultado do tratamento; Reabilitação bucal. 


\begin{abstract}
Facial fractures are a public health problem due to the need for specialized care, adequate environment and financial resources. They have different causes, and sports, work, traffic accidents and falls stand out as the main ones, being the same related to age, gender of the patient and direction of the trauma. This paper aims to report the clinical case of a male patient who fell 4.5 meters high, pushing his face against the edges of a swimming pool. Attended at the Maria Aparecida Pedrossian University Hospital, requiring outpatient and surgical care for the treatment of multiple facial fractures. The initial physical examination showed edema in the middle and lower thirds of the face, preserved visual acuity and ocular motility, mobility in the parasymphysis, laceration in the lower lip, marked mobility in dental elements, fragments of exposed oral bone tissue in the premaxilla region, absence of altered teeth and mandibular movements. Computed tomography showed a comminuted fracture in the anterior maxilla, bilateral fracture of the condyle and fracture of the mandibular parasymphysis on the left side. Thus, the treatment of choice was surgery under general anesthesia for placement of the Erich bar and maxillomandibular block, reduction and fixation of anterior mandible fractures and removal of bone fragments and unsupported teeth in the maxillary region. In the postoperative controls, the patient remained without complaints after the blockage was removed, with preserved mandibular movements and return to masticatory functions. Six months after the trauma, the patient is fully rehabilitated.
\end{abstract}

Keywords: Fractures, bone; Therapeutics; Treatment outcome; Mouth rehabilitation.

\title{
Resumen
}

Las fracturas faciales son un problema de salud pública debido a la necesidad de atención especializada, ambiente adecuado y recursos económicos. Tienen diferentes causas, y se destacan como principales los accidentes deportivos, laborales, de tránsito y las caídas, estando las mismas relacionadas con la edad, el sexo del paciente y la dirección del traumatismo. Este trabajo tiene como objetivo reportar el caso clínico de un paciente masculino que cayó desde 4,5 metros de altura, empujando su rostro contra los bordes de una piscina. Acudió al Hospital Universitario María Aparecida Pedrossian, requiriendo atención ambulatoria y quirúrgica para el tratamiento de múltiples fracturas faciales. El examen físico inicial mostró edema en los tercios medio e inferior de la cara, agudeza visual y motilidad ocular conservadas, movilidad en la parasínfisis, laceración en el labio inferior, marcada movilidad en elementos dentarios, fragmentos de tejido óseo oral expuesto en la región de la premaxila, ausencia de dientes alterados y movimientos mandibulares. La tomografía computarizada mostró una fractura conminuta en la región anterior del maxilar, fractura bilateral del cóndilo y fractura en la parasínfisis mandibular del lado izquierdo. Así, el tratamiento de elección fue la cirugía bajo anestesia general para colocación de barra de Erich y bloqueo maxilomandibular, reducción y fijación de fracturas mandibulares anteriores y extracción de fragmentos óseos y dientes no soportados en la región maxilar. En los controles postoperatorios el paciente se mantuvo sin quejas luego de removida la obstrucción, con movimientos mandibulares conservados y retorno a las funciones masticatorias. Seis meses después del trauma, el paciente está completamente rehabilitado.

Palabras clave: Fracturas óseas; Terapéutica; Resultado del tratamiento; Rehabilitación bucal.

\section{Introdução}

As fraturas faciais afetam em maior número indivíduos do sexo masculino, entre a segunda e terceira década de vida. (D’Avila, et al., 2015; Minari, et al., 2020; Samierad et al., 2015; Schneider et al., 2015), sendo a mandíbula, em virtude de sua proeminência e articulação, o osso mais cometido. As causas das fraturas mandibulares são multifatoriais, incluindo os acidentes esportivos e de trabalho, agressões físicas e acidentes automobilísticos. Destas, a área mais comumente afetada é a região condilar, seguida por fratura de corpo, ângulo e sínfise (Ribeiro et al., 2012).

As fraturas de côndilo, ou cabeça da mandíbula, além de serem as mais comuns, são as que apresentam o maior número de controvérsias quanto à escolha de seu tratamento e maior dificuldade de diagnóstico, sendo de suma importância exames de imagem em consonância com acurado exame clínico, para indicação de um tratamento cirúrgico ou conservador que devolva a função mandibular primária (Manganello et al., 2002).

A avaliação do paciente com trauma facial é de suma importância para a escolha do tratamento, sendo o primeiro passo a estabilidade sistêmica, como o controle dos sinais vitais, passagem livre das vias aéreas e estado neurológico. Durante a avaliação inicial, todos os problemas que possam colocar a vida do paciente em risco, devem ser sanados (Zarpellon et al., 2015; Hupp et al., 2015; Leporace et al., 2009; Manganello et al., 2002).

Para Hayward e Scott (1993) além da idade do paciente, a localização da fratura e o grau de deslocamento da mesma são relevantes na escolha da terapêutica. A maioria dos traumas condilares, são tratados de forma incruenta, conservadora, por 
meio de bloqueio maxilomandibular (BMM), associado a fisioterapia elástica e dieta líquida. Mesmo na presença de desvio de côndilo, tendem à abordagem conservadora, enquanto fraturas com luxação, tendem a indicação cirúrgica.

No auxílio, a confirmação da fratura são realizados exames complementares, como os exames de imagem. As radiografias podem trazer clareza a um exame clínico, e determinar com maior precisão a extensão da fratura. A tomografia computadorizada é o exame "padrão-ouro" (Teles et al., 2016; Tanrikulu et al., 2001), frequentemente utilizado pelos profissionais, visto que sua imagem permite uma noção tridimensional do arcabouço facial, suplementando o diagnóstico das fraturas (Lee et al., 1987).

No tocante ao tratamento, ele varia desde uma fixação maxilomandibular, dito conservador, feito com barras fixadas na arcada dentária superior e inferior, ligadas entre si com fios metálicos, até uma abordagem cirúrgica cruenta, com auxílio de sistemas de placas e parafusos de fixação (Gaetti-Jardim et al., 2014), a depender do caso.

A redução fechada, com BMM visa restabelecer a oclusão prévia ao trauma e, em certos casos, pode estabilizar suficientemente o osso para permitir a sua reparação tecidual. Está indicada quando a linha de fratura e o vetor de tração muscular são favoráveis e mantêm a fratura adequadamente reduzida, em casos que o paciente tem uma dentição saudável, com dentes suficientes para obter uma oclusão estável (Goodday et al., 2013).

$\mathrm{Na}$ escolha do tratamento, é primordial o restabelecimento da oclusão e função do sistema estomatognático, para que haja o mínimo ou nenhuma sequela estética e funcional ao paciente, auxiliando sua reintegração em meio à sociedade (Marcolino, 2014), pois quando mal abordados, essas sequelas podem gerar marginalização do indivíduo no convívio social.

Desta feita, é objetivo apresentar um caso clínico de paciente vítima de trauma em face após queda de grande altura, resultando em múltiplas fraturas, tratado de forma cirúrgico-conservadora.

\section{Metodologia}

O presente caso trata-se de um relato com fins descritivos, exploratórios e com abordagem qualitativa, a fim de destacar sua relevância clínica e fomentar pesquisas e novos relatos baseados sempre em evidência científica, tendo por base Pereira (2018) e Souza et al. (2021). O paciente consentiu por escrito o uso de suas imagens e de apresentação do seu caso clínico no meio científico.

Deste modo, paciente do sexo masculino, 22 anos de idade, compareceu ao Pronto Atendimento Médico do Hospital Maria Aparecida Pedrossian (HUMAP) da Universidade Federal de Mato Grosso do Sul (UFMS), via sistema de regulação de vagas do Sistema Único de Saúde, com histórico de queda de andaime de cerca de 4,5 metros de altura, cursando com trauma em face. Apresentou-se a primeira consulta em bom estado geral, eupneico, deambulando e verbalizando normalmente.

Durante anamnese, referiu acidente de trabalho, com queda após se desequilibrar, impelindo face na borda de uma piscina. No momento do trauma referiu avulsão de dentes anteriores e alteração da oclusão dentária. Negou comorbidades sistêmicas, assim como uso drogas ilícitas, tabagismo, etilismo e alergias medicamentosas.

$\mathrm{Na}$ avaliação física, apresentou assimetria facial às custas de edema em terço médio e inferior da face, com laceração em lábio inferior que já havia sido suturado em seu município de origem. No exame intraoral, observou-se discreta limitação de abertura bucal, alteração oclusal, edema e equimose em lábio superior bem como ausência dos elementos dentários 11 e 22, 21, 12 e, o elemento 13 com grande mobilidade; além de, laceração de mucosa gengival na região do dente 12, crepitação em parassínfise mandibular do lado esquerdo e equimose em assoalho de boca.

Em análise de exames complementares, foi solicitado radiografia de tórax, sendo descartada a presença de corpo estranho no pulmão; solicitação de bioquímicos e tomografia computadorizada de face, apresentando nos cortes coronal, axial e sagital gerando o diagnóstico de fratura cominutiva em região anterior de maxila, fratura bilateral de cabeça da mandíbula e em parassínfise mandibular do lado esquerdo. 
Assim, em virtude do quadro, realizou-se sob anestesia local, a limpeza dos ferimentos e sutura dos alvéolos, seguido por redução de traumatismo dentoalveolar e estabilização da oclusão e da fratura condilar, com barra de Erich e fio de aço. Além disso, fez-se o planejamento cirúrgico para realização da redução da fratura de parassínfise mandibular e fratura em região de pré-maxila, sob anestesia geral, em um segundo tempo cirúrgico (Figura 1).

Figura 1: Aspectos clínico e imaginológico do paciente. Múltiplas fraturas faciais: cominuição da pré-maxila, fratura parassinfisária a esquerda e da cabeça da mandíbula bilateralmente.

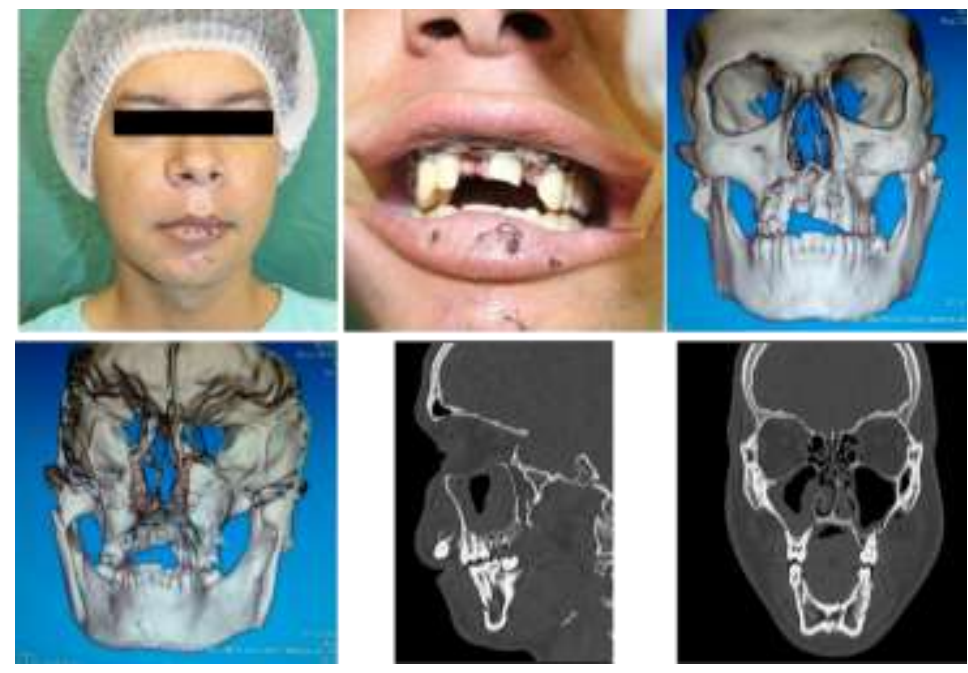

Fonte: Autores.

No sétimo dia de internação, o paciente foi submetido a procedimento de redução e osteossíntese em fratura anterior da mandíbula, com placas e parafusos de titânio, desbridamento de tecidos inviáveis em região anterior da maxila, limpeza rigorosa realizada com gaze e antisséptico (Iodopovidona 10\%), remoção de tecido ósseo cominuído nas regiões de alveólos dos dentes $13,12,11,21$ e 22, e manutenção da barra de Erich, superior e inferior para bloqueio rígido maxilomandibular para tratamento conservador de fratura condilar bilateral.

Ao exame tomográfico do pós-operatório imediato, foi observado posicionamento satisfatório dos materiais de osteossíntese bem como das fraturas fixadas. (Figura 2). 
Figura 2: Aspectos imaginológicos pós-operatório. Dispositivos de síntese óssea em posição. Posicionamento satisfatório das cabeças da mandíbula.
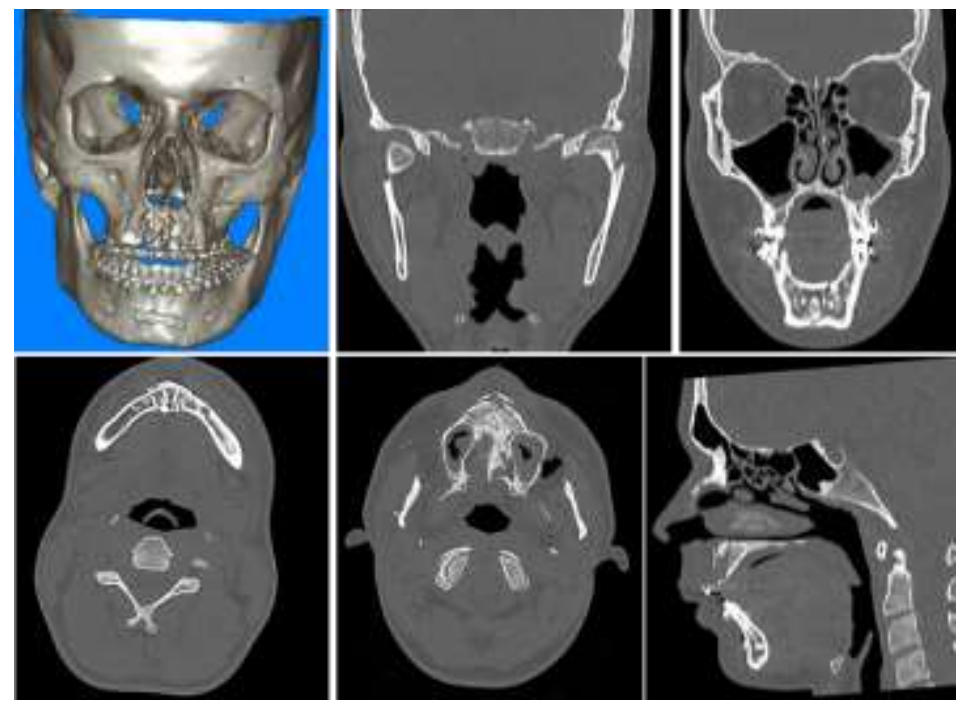

Fonte: Autores.

Em pós-operatório de 24 horas, o paciente apresentava-se em bom estado geral, com edema em face compatível com o procedimento cirúrgico realizado, bloqueio maxilomandibular em posição, ausência de sangramento intraoral e sem quaisquer sinais flogísticos, seguindo em alta hospitalar com prescrição medicamentosa e orientado quanto aos cuidados pós-operatórios, para limpeza rigorosa dos remanescentes dentários, dieta liquidificada e uso das medicações prescritas (amoxicilina 500mg de $8 / 8 \mathrm{~h}$ por 7 dias, ibuprofeno $600 \mathrm{mg}$ de $12 / 12 \mathrm{~h}$ por 3 dias, dipirona $500 \mathrm{mg}$ de $6 / 6 \mathrm{~h}$ por 2 dias e solução oral de clorexidina $0,12 \%$ de $12 / 12 \mathrm{~h}$ por 14 dias).

Em retorno ambulatorial após 07 dias do procedimento, o paciente relatou não apresentar sintomatologia dolorosa, apenas dificuldade para se alimentar, resultado do uso do BMM. As suturas se apresentavam em posição, assim como o bloqueio maxilomandibular.

Quinze dias após o procedimento cirúrgico, o paciente foi novamente avaliado e orientado quanto à necessidade de higiene oral, dificultada com a presença do BMM. Todavia, com boa recuperação do procedimento cirúrgico e oclusão dental reestabelecida, compatível com relatos do próprio paciente, foi preconizado bloqueio maxilomandibular com auxílio de elásticos por mais 30 dias, para manutenção da oclusão e, foi instituída fisioterapia de abertura, fechamento, protrusão e retrusão da mandíbula.

Decorridos 45 dias da instalação do BMM, o mesmo foi removido e o paciente foi encaminhado ao cirurgião-dentista para adequação e reabilitação oral, com retorno agendado para acompanhamento evolutivo do quadro. Em retorno subsequente de 60 dias, ao exame físico extraoral, apresentou boa cicatrização dos ferimentos de face. No exame intraoral apresentava prótese parcial removível em dentes anteriores da maxila, bom aspecto cicatricial da mucosa e ausência de sinais inflamatórios (Figura $3)$. 
Figura 3: Pós-operatório de 60 dias. Paciente com reabilitação protética devolvendo estética e função mastigatória.
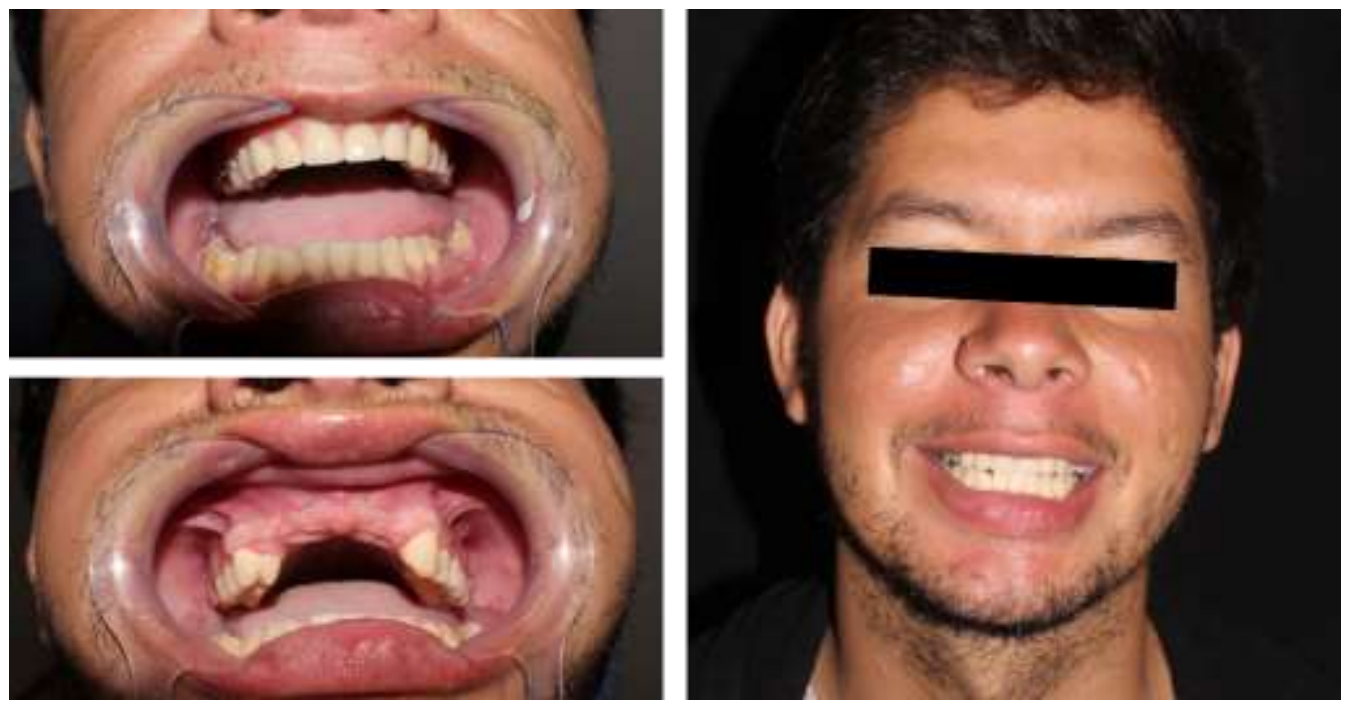

Fonte: Autores.

\section{Discussão}

As fraturas faciais apresentadas pelo caso acima foram consideráveis, porém, se tornam menores quando colocadas diante ao histórico de queda de 4,5 metros de altura e o fato de que o paciente não teve fratura em outra região do corpo, ou mesmo, um traumatismo cranioencefálico, comum em casos de quedas de grande altura, quando há grande força externa envolvendo região de cabeça e pescoço, com sintomas que podem se apresentar de imediato ou tardios, incluindo, complicações secundárias das lesões na cabeça decorrentes de impactos na região Maxilofacial que podem até levar a óbito (Joshi et al., 2018; Pappachan \& Alexander, 2012). Sendo assim, mesmo que a perda estética e comprometimento momentaneamente funcional do paciente, supõe-se que o quadro foi irrisório ante as consequências que poderiam ter sido geradas.

Ainda assim, corrobora-se com muitos estudos comparativos, onde há uma tendência de maior prevalência de traumas faciais em jovens, com idade média de 20 a 30 anos, do sexo masculino (Correia et al., 2011; O'connor et al., 2015; Park et al., 2015; Samieirad et al., 2015; Schneider et al., 2015; Zaleckas et al., 2015; Zhou et al., 2016), mostrando-se como os grupos mais afetados, propensos a traumatismos faciais, principalmente quando em caráter de múltiplas fraturas (Park et al., 2015; Samieirad et al., 2015; Schneider et al., 2015).

Fraturas com ou sem desvio, sem luxação de côndilo, tendem a abordagem conservadora, como descrito no caso, com auxílio de um bloqueio maxilomandibular, a fim de restabelecer a oclusão e estabilizar suficientemente o osso, para permitir a sua reparação, respaldando o tratamento de escolha do caso relatado neste trabalho (Manganello et al., 2002); Goodday et al., 2013).

Assim, o tratamento conservador das cabeças mandibulares, baseou-se no bloqueio rígido nos 15 dias iniciais, e após, manutenção de bloqueio com elástico por 30 dias (Manganello et al., 2002). Escolha desafiadora frente às inúmeras fraturas descritas bem como a má oclusão do paciente previamente a queda, que poderia comprometer todo o tratamento e agravar àquelas, resultando em um deslocamento das mesmas, por conta da oclusão instável (Rampaso et al., 2012; Goodday et al., 2013).

Em região de sínfise mandibular, foi realizado tratamento em campo aberto, com utilização de apenas uma placa, sistema 2.0mm (Meneses et al., 2020; Miloro et al., 2016). Em região anterior de maxila, demonstrou a necessidade de redução aberta em centro cirúrgico, sob anestesia geral, para remoção de fragmentos ósseos com mobilidade, sem possibilidade de fixação em virtude da cominuição do local (Miloro et al., 2016). Ao se realizar a limpeza rigorosa e desbridamento da região, fez-se a 
remoção de fragmentos ósseos soltos que poderiam comprometer a cicatrização local e atuar futuramente como corpo estranho, propiciando o desenvolvimento de infecção pela ausência de nutrição sanguínea no mesmo. Seguindo o mesmo princípio, por conta da falta de suporte ósseo, não foi possível manter os dentes 13, 12, 11, 21 e 22.

Em contrapartida, a literatura salienta que os pacientes tratados com redução aberta tendem a ter menores complicações em comparação com a redução fechada de fraturas mandibulares, além de um menor tempo de tratamento e um retorno mais rápido às funções mastigatórias (Chrcanovic, 2013). Contrapondo, o relato de caso deste trabalho, em que o paciente foi tratado com redução fechada no tratamento bilateral de côndilo, obteve-se resultados satisfatórios, com retorno de funções mandibulares, limitadas inicialmente, mas com retorno à normalidade em 45 dias e, manutenção da integridade de estruturas nobres faciais uma vez que a redução aberta também possui seus riscos, como lesão de estruturas nobres, vasos próprios da região, nervos, e infecção pós-operatória. O mesmo autor ainda menciona que na ausência de estabilidade oclusal o BMM e a redução fechada ou tratamento conservador levam a resultados satisfatórios. Tal terapêutica vai de encontro a Soares et al. (2019), que advogam nos casos de fratura parassinfisária e de côndilos mandibulares o BMM como opção viável, sobretudo, quando associado a fisioterapia fonoaudióloga intensiva.

O sucesso terapêutico é determinado pela recuperação das funções faciais (Teles et al., 2016), sendo que no caso relatado, há abordagem de fraturas múltiplas da face, onde a proximidade das estruturas faciais com a região intracraniana e o fato de a face ser uma região de grande exposição quando comparadas as outras, faz com que fraturas nesta área tenham grande relevância em relação a estética e também levando em consideração o impacto, que podem originar sequelas permanentes, e até à morte do paciente (Batstone et al., 2007; Pappachan \& Alexander, 2012). No caso em questão, em virtude da gravidade quando se considera a altura da queda, é relevante o fato que não tenha sofrido sequelas ou traumas mais graves, como traumatismo cranioencefálico (Gaudêncio et al., 2013).

A literatura é vasta. A escolha do melhor método de tratamento deve ser feita caso a caso e de acordo com a experiência do cirurgião (Péder et al., 2020). O tratamento de fraturas faciais é desafiador, uma vez que é essencial devolver qualidade de vida com retorno funcional ao paciente. Em síntese, por se tratar de um caso peculiar, onde o quadro do paciente poderia ter sido de grandes sequelas ou até mesmo ter o levado a morte, dispomos de uma situação, onde apesar de fraturas múltiplas da face, as consequências foram mínimas, para o quadro, considerando o que poderia ter acontecido, corroborando com informações descritas em Carvalho, et al. (2010). Após 60 dias pós-operatório, paciente seguiu de alta pela especialidade de cirurgia e traumatologia bucomaxilofacial, sendo encaminhado para continuação do tratamento estético e reabilitador, com cirurgião dentista em ambiente clínico, obtendo resultados satisfatórios estético-funcionais.

\section{Considerações Finais}

Ainda que limitado por ser um relato de caso, necessitando de maior número de casos para gerar uma confiabilidade estatística e demonstrar a credibilidade às técnicas empregadas, este relato desafiador e complexo, tanto na sua dificuldade técnica, quanto na magnitude do agente causador do trauma facial, mostrou bons resultados.

O fato de o paciente não ter tido consequências muito mais severas do que as apresentadas, já configurou sucesso e a escolha de técnicas mais conservadoras na terapêutica empregada demonstrou estar em consonância com este sucesso. O paciente se encontra bem e reabilitado, com retomada das atividades laborais e de sua qualidade de vida.

\section{Agradecimentos}

O presente trabalho foi realizado com apoio da Fundação Universidade Federal de Mato Grosso do Sul -UFMS/MEC -Brasil. 


\section{Referências}

Batstone, M. D., Monsour, F. N., Pattel, P., \& Lynham, A. (2007). The patterns of facial injury suffered by patients in road traffic accidents: a case controlled study. International Journal of Surgery. 5: 250-4.

Carvalho, M. F., Herreo, R. K. R., Moreira, D. R., Urbano, E. S., \& Reher, P. (2010). Princípio de Atendimento Hospitalar em Cirurgia Buco-MaxiloFacial. Revista Cirurgia Traumatologia Buco-Maxilo-Facial, Camaragibe. 10(4): 79-84.

Chrcanovic, B. R. (2013). Open versus closed reduction: Comminuted mandibular fractures. Oral and Maxillofacial Surgery. 17(2), 95-104. https://doi.org/10.1007/s10006-012-0349-2.

D’avila. S., Barbosa, K. G. N., Bernardino, I. M., Nobrega, L. M., Bento, P. M., \& Ferreira, E. F. (2015). Facial Trauma Among Victims of Terrestrial Transport Accidents. Brazil Journal Otorhinolaryngoly. Rio de Janeiro. 82(3): 314-320.

Gaudêncio, T. G., \& Leão, G. de M. (2013). A Epidemiologia do Traumatismo Crânio- Encefálico: Um Levantamento Bibliográfico no Brasil. Revista Neurociências. 21(3), 427-434. 2013, v.21, 8261.

Gaetti-Jardim, E. C., Silva, H. C. L., Pereira, T. T. M., Masocatto, D. C., Oliveira, M. M., \& Mendonça, J. C. G. (2014). Tratamento de fratura complexa de terço médio de face associada a ferimento extenso. Archives of Health Investigation. 3(3):1-7.

Goodday, R. H. Management of fractures of the mandibular body and symphysis. 2013. Oral and Maxillofacial Surgery Clinics of North America. 25(4):60116. 10.1016/j.coms.2013.07.002.

Hayward, J., \& Scott, R. A. (1993). Fractures of mandibular condyle. Journal of Oral and Maxillofacial Surgery. 1:57-61.

Hupp, J. R., Ellis III, E., \& Tucker, M. R. (2015). Cirurgia Oral e Maxilofacial Contemporânea. (6a ed.), Elsevier; 2015.

Joshi, U. M., Ramdurg, S., Saikar, S., Patil, S., \& Shah, K. (2018). Brain Injuries and Facial Fractures: A Prospective Study of Incidence of Head Injury Associated with Maxillofacial Trauma. Journal of Maxillofacial and Oral Surgery. 17(4):531-537.

Lee, K. F., Wagner, L. K., Lee, Y. E., Suh, J. H., \& Lee, S. R. (1987). The impact-absorbing effects of facial fractures in closed-head injuries. An analysis of 210 patients. Journal of Neurosurgery. 66: 542- 7.

Leporace, A. A. F., Paulesini Júnior, W., Rapoport, A., E. C. B. C., \& Denardin, O. V. P. Estudo epidemiológico das fraturas mandibulares em hospital público da cidade de São Paulo. 2009. Revista do Colégio Brasileiro de Cirurgiões. 36(6): 472-477.

Manganello, L. C., \& Silva, A. A. F. (2002). Manidbular condylar fractures: classification and treatment. Revista Brasileira de Otorrinolaringologia. 68(5): 749-55.

Marcolino, P. R. B. (2014). Ressocialização das vítimas de fratura de face. Revista de Cirurgia e Traumatologia Buco-maxilo-facial. 14(3): 85-90.

Mendonça, J. C. G., Gaetti-Jardim, E. C., Manrique, G. R., Lopes, H. B., \& Freitas, G. P. (2013). Acesso Cirúrgico para Tratamento de Fraturas Mandibulares: Revisão de Literatura. Archives of Health Investigation. 2(2):19-23.

Meneses-Santos, D., Oliveira, M. M. M., Sol, I., \& Rodrigues, C. (2020). Tratamento cirúrgico de fratura cominutiva de mandíbula envolvendo simplificação com miniplacas: Relato de dois casos. Research, Society and Development. 9(12): e43991211398.

Miloro, M., Waite, P. D., Larsen, P. E., \& Ghali, G. E. (2016). Principios de cirurgia Bucomaxilofacial de Peterson. 3. ed. São Paulo: Santos Editora. 1344 p.

Minari, I. S., Figueiredo, C. M. B. F., Oliveira, J. C. S., Brandini, D. A., \& Bassi, A. P. F. (2020). Incidencia de multiplas fraturas faciais: estudo retrospective de 20 anos. Research, Society and Development. 9(8): e32795347.

Nahum, A. M. (1975). The biomechanics of maxillofacial trauma. Clinics in Plastic Surgery. 2(1):59-64.

O'Connor, R. C., Shakib, K., Brennan, P. A. (2015). Recent advances in the management of oral and maxillofacial trauma. British Journal of Oral and Maxillofacial Surgery. 53(10):913-21.

Pappachan, B., \& Alexander, M. (2012). Biomechanics of cranio-maxillofacial trauma. Journal of Maxillofacial and Oral Surgery. 11: 224-30.

Park, K. P., Lim, S. U., Kim, J. H., Chun, W. B., Shin, D. W., Kim, J. Y., \& Lee, H. (2015). Fracture patterns in the maxillofacial region: a four-year retrospective study. Journal of the Korean Association of Oral and Maxillofacial Surgery. 41: 306-16.

Péder, S. N. S., Rodriguez, E. A. S., Quinto, J. H. S., et al. (2020). Conservative treatment of mandibular condo fracture after firearm injury: inter-relationship between surgery and orthodontics. Orthodontic Science and Practice. 13(50):70-75.

Pereira, P. A. 2018. Metodologia da pesquisa científica. UFSM.

Ribeiro, F. F., Hilgert, R., Soares, L. Y., Magnabosco Neto, A. E., Santangelo, A. G., Pacher, G. T., \& Fonseca, L. A. M. (2012). Estudo da prevalência de fraturas mandibulares em Joinville- SC. Revista Cirurgia e traumatologia buco-maxilo-facial. 12(3): 79-84.

Samieirad, S., Tohidi, E., Shahidi-Payam, A., Hashemipour, M. A., \& Abedini, A. (2015). Retrospective study maxillofacial fractures epidemiology and treatment plans in Southeast of Iran. Medicina Oral, Patologia Oral, Cirugia Bucal. 20: 729-36.

Schneider, D., Kämmerer, P. W., Schön, G., Dinu, C., Radloff, S., \& Bschorer, R. (2015). Etiology and injury patterns of maxillofacial fractures from the years 2010 to 2013 in Mecklenburg-Western Pomerania, Germany: A retrospective study of 409 patients. Journal of Cranio-Maxillofacial Surgery. 43(10):1948-51. 
Research, Society and Development, v. 11, n. 2, e23511225639, 2022

(CC BY 4.0) | ISSN 2525-3409 | DOI: http://dx.doi.org/10.33448/rsd-v11i2.25639

Silva, J. J. S., Lima, A. A. A., Dantas, T. B., Frota, M. H. A., Parente, R. V., \& Lucena, A. L. S. P. N. (2011). Fraturas de Mandíbula: Estudo Epidemiológico de 70 Casos. Revista Brasileira de Cirurgia Plástica. 26(4): 645-648.

Soares, L. O., Carvalho, B. C. S., Resende, R. G., \& Gomes, A. L. (2019). Tratamento fechado em fratura mandibular: parassinfisária e côndilar bilateral - relato de caso clínico. Revista da Faculdade de Odontologia, Universidade de Passo Fundo. 24(2): 292-298.

Souza, Y. D., Seco, A. J. L. G., Silva, J. C. L. Da., Pereira, K. F. S., \& Jardim, E. C. G. (2021). Kaposi’s Sarcoma in an HIV and Covid-19 positive patient. Research, Society and Development.10(11), e19101119149.

Tanrikulu, R., \& Erol, B. (2001). Comparison of computed tomography with conventional radiography for midfacial fractures. Dentomaxillofacial Radiology. 30(3): $141-146$

Teles, I. C. M., Cruz, J. A., Parreira, D. R., Sousa, G. H. C., \& Curvina, M. M. G. (2016). Fratura panfacial: um relato de caso. Revista de Medicina e Saúde de Brasília. 5(3): 222-8.

Zaleckas, L., Pečiulienè, V., Gendvilienè, I., Pūrienè, A., \& Rimkuvienė, J. (2015). Prevalence and etiology of midfacial fractures: a study of 799 cases. Medicina (Kaunas). 51: 222- 7 .

Zarpellon, A. T., Cruz, G. O. A., Gus, I. O., Moreira, G. H. G., Chamma, R. S., \& Masi, F. D. J. (2015). Revisão de 150 casos de fratura de mandíbula entre os anos de 2010 e 2013 no Hospital Universitário Cajuru - Curitiba, PR. Revista Brasileira Cirurgia Plástica. 30(4): 609-614.

Zhou, H., Lv, K., Yang, R., Li, Z., \& Li, Z. (2016). Mechanics in the Production of Mandibular Fractures: A Clinical, Retrospective Case-Control Study. PLoS One. 11: $\mathrm{e} 0149553$ 